\title{
Patient Reported Outcome Measures After Breast Augmentation - Using the BREAST-Q IS
}

\author{
Randi T Jayasinghe ${ }^{\prime}$ \\ Rasa Ruseckaite (1D) \\ Pragya Gartoulla' \\ Elisabeth Elder ${ }^{2}$ \\ Ingrid Hopper'
}

'Department of Epidemiology and Preventive Medicine, School of Public Health and Preventive Medicine, Monash University, Melbourne, Victoria, Australia; ${ }^{2}$ Westmead Breast Cancer Institute and Breast Surgeons of Australia and New Zealand, Westmead, NSW, Australia
Correspondence: Ingrid Hopper Department of Epidemiology and Preventive Medicine, School of Public Health and Preventive Medicine, Monash University, 553 St Kilda Road, Melbourne,

Victoria, 3004, Australia

Tel +61 399030569

Email ingrid.hopper@monash.edu
Purpose: Cosmetic breast augmentation procedures are commonly performed breast device surgeries. The Australian Breast Device Registry (ABDR) administers a five-question patient reported outcome measure (PROM), the BREAST-Q Implant Surveillance module (BREAST-Q IS), to patients 1,2 and 5 years after breast device surgery. The measure includes an open-ended question to add any comments. This study aimed to use the responses to this open-ended question to assess participants' experiences of breast devices 1 and 2 years after breast augmentation. The secondary objective was to identify emerging and important issues relating to breast augmentation and devices.

Patients and Methods: This qualitative descriptive study was conducted using a randomly selected sample of 268 responses to the open-ended question in the BREAST-Q IS, from the ABDR database. These responses were from patients who underwent breast augmentation between 2015 and 2018. Comments were analyzed using conventional content analysis in NVivo 12.

Results: Four major themes were identified: satisfaction following breast augmentation, dissatisfaction following breast augmentation, complications and breast symptoms following breast augmentation and other comments. Two dominant themes were regarding satisfaction $(\mathrm{n}=112)$ with overall surgical outcome, medical team, and post-operative appearance and complications and breast symptoms $(n=177)$ following breast augmentation. Emerging issues identified were rippling of breast implants and breast implant illness (BII).

Conclusion: PROMs can be used to understand patients' perspectives on various aspects of their own surgical experiences. Participants provided responses regarding complications and breast symptoms experienced, and rippling of the breast implants and BII are emerging issues after breast augmentation.

Keywords: patient reported outcome measures, breast augmentation, Breast-Q Implant Surveillance, breast device surgery, Australian Breast Device Registry

\section{Introduction}

Breast device surgeries are increasingly popular. In Australia, an estimated 20,000 women undergo breast device surgery each year, ${ }^{1}$ of which approximately $75 \%$ is cosmetic breast surgery, and the remainder is reconstruction after breast cancer or to correct developmental abnormalities. ${ }^{1,2}$ Breast devices, which include breast implants, tissue expanders and dermal matrices and meshes, are classified as class III (high risk) implantable medical devices for the potential health risks they pose for patients. ${ }^{3}$ Breast implants have been associated with a number of health scares, ${ }^{4}$ the most recent being Breast Implant Associated Anaplastic Large Cell Lymphoma (BIA-ALCL). ${ }^{5}$ Breast implants are not considered to be lifetime devices and at least $30 \%$ of breast implant procedures are revision surgeries of previous implants. ${ }^{6}$ 
Breast implants are implantable gel or fluid filled pouches that are inserted in the breast area in order to improve or form the shape of a breast. $^{7}$ Tissue expanders are implanted after mastectomy and serially expanded to form a pocket and then replaced with a permanent breast implant. Dermal mesh and matrices are tissue substitutes. Dermal matrix is human skin of which the epidermis and the dermis has been removed leaving an extracellular matrix. ${ }^{8}$ This matrix is used as a scaffold on which patient's own cells grow and revascularize creating an extra layer of tissue. ${ }^{8,9}$ Synthetic meshes made from absorbable and permanent fibres are also used in breast device surgery and they provide support to maintain the shape of the breasts. ${ }^{9}$

Ongoing post-market surveillance is required to ensure the safety of breast devices. The Australian Breast Device Registry (ABDR) is a national clinical quality registry which performs post-market surveillance of breast devices and can be used to assess the quality of breast device surgery as well. ${ }^{10}$ One method currently being used by the ABDR to assess breast device performance is Patient Reported Outcome Measures (PROMs). PROMs come directly from patients without the interpretation by a clinician or anyone else, ${ }^{11,12}$ and have the potential to enhance quality and safety, and improve healthcare by placing patients at the center of decision-making. ${ }^{13}$ The use of PROMs have extended beyond clinical research and into clinical care, including clinical registries and quality improvement activities as well. ${ }^{7,10,13}$

The ABDR uses the BREAST-Q Implant Surveillance module (BREAST-Q IS), which is a novel instrument designed for use by breast device registries with the specific purpose of monitoring breast devices by detecting a potential signal earlier than waiting for revision surgery to occur. ${ }^{7}$ The BREAST-Q IS was derived from the full bank of questions from the BREAST-Q PROM, and includes five questions relating to look, feel, rippling, pain and tightness that were found to be most sensitive for detecting a poorly performing breast devices. ${ }^{7,14}$ The BREAST-Q IS also includes an open-ended question inviting patients to comment about their experience of breast devices after undergoing surgery. ${ }^{3,7}$

The current study aimed to explore the comments offered by patients in the open-ended question of the BREAST-Q IS module relating to breast augmentation surgery. We aimed to understand the perspectives of patients regarding their experiences of breast devices and identify emerging issues relating to breast device surgery and breast devices in cosmetic breast augmentation surgery.

\section{Patients and Methods}

The ABDR is a national clinical quality registry, funded by the Australian Government and established in $2015 .^{3}$ The registry invites plastic surgeons, general/breast surgeons and cosmetic surgeons, and after agreeing to participate, they are expected to contribute data on all of their patients at the time of primary operation or revision/return to the theatre. The ABDR uses an "opt-out" approach to patient consent. For active post-market surveillance, the BREAST-Q IS is sent via a text message with a unique weblink for each participant to complete the questionnaire. ${ }^{2}$ If no reply is received, various other methods were used to collect the data including email, phone calls and post. At the end of 2019, 563 surgeons, 271 sites and 49,563 patients were participating in the ABDR. ${ }^{1}$

\section{Data Collection and Processing}

For this analysis, open text responses provided by participants who were registered in the ABDR were used. Participants were included if they were 18 years and older and had undergone breast augmentation surgery between 2015 and 2018, completed BREAST-Q IS at either follow up year 1 and/or follow up year 2 and commented on the free comment section and had not opted out of the registry or follow up. This study was approved by the Monash University Human Ethics Research Committee, Melbourne, Australia (Project ID:23939).

Data extraction, processing and analysis occurred between April 2020 and July 2020. The number of PROMs data that were available at the time of data extraction was 33,847 , and 16,850 participants had responded to the open-ended question. All responses with missing answers to the open-ended questions were removed $(n=16,997)$. A sample of 500 deidentified responses to PROMs were randomly extracted relating to participants who had received breast augmentation and reconstruction between years 2015-2018. This included 232 responses from participants who received a breast reconstruction and 268 responses from participants who underwent breast augmentation. For the purpose of this study, we only reported on the 268 responses provided by participants who had received a breast augmentation procedure.

\section{Data Analysis}

All data were aggregated, meaning that a participant may not have provided responses at both follow up year 1 and 2. The extracted PROMs data were sorted and filtered using Microsoft Excel 2020 before importing to NVivo 12 software for analysis. The responses were categorized by the follow-up year (either 1 year or 2 years) and further 
stratified by age into each of the following age categories: 18 to 30 years, 31 to 50 years and $\geq 51$ years. A qualitative approach was used to understanding participants' experiences following breast augmentation and identifying emerging issues through participants' subjective responses. ${ }^{15}$ Conventional content analysis was performed, including open coding and axial coding to identify themes. ${ }^{16,17}$

A theme is a concept, attribute or descriptor that brings repeating ideas together. It is an implicit element, and its underlying meaning is discovered through interpreting the data. ${ }^{18}$ Themes may also contain sub-themes to gain a comprehensive understanding of the data and discover any patterns in participants' responses. ${ }^{18}$ In our study we were able to derive both themes and sub-themes to gain an understanding of patients' experience following breast augmentation.

The consolidated criteria for reporting qualitative research (COREQ) 32-item checklist used for explicit reporting of the study methods. ${ }^{19}$

\section{Results}

\section{Study Participants}

A total of 268 open-text responses, provided by 261 participants who had undergone breast augmentation between 2015 and 2018 and registered in the ABDR were analyzed, with 7 participants filling out the BREAST-Q IS at both follow up year 1 and 2 . The mean age of the participants was 38 (SD 12) years (Table 1).

\section{Themes Identified}

The following four major themes were identified from the open text comments section of the BREAST_Q IS: 1) Satisfaction with breast augmentation and 2) Dissatisfaction with breast augmentation, 3) Complications and breast

Table I Follow Up Year and Age at Operation of Breast Augmentation Participants $(\mathrm{n}=268)^{*}$

\begin{tabular}{|l|l|l|}
\hline \multirow{2}{*}{} & \multicolumn{2}{|c|}{ Follow Up } \\
\cline { 2 - 3 } & Year I (n=37) & Year 2 (n=23I) \\
\hline \multicolumn{2}{|l|}{ Age at operation (years) } & 76 \\
\hline $18-30$ & 7 & 123 \\
\hline $31-50$ & 22 & 32 \\
\hline$>51$ & 8 & \multicolumn{2}{|c|}{} \\
\hline
\end{tabular}

Note: $* 7$ participants commented on both follow up years $I$ and 2.

Abbreviations: ABDR, Australian Breast Device Registry; PROMs, Patient Reported Outcome Measures; BREASTQ-IS, BREAST-Q Implant Surveillance; BII, Breast Implant IIIness; BIA-ALCL, Breast Implant Associated Anaplastic Large Cell Lymphoma. symptoms following breast augmentation and 4) Other comments on aspects that are not directly related to outcome of breast augmentation.

\section{Satisfaction with Breast Augmentation}

\section{The Overall Surgical Outcome and Implants}

Participants $(n=76)$ reported that they were satisfied with the decision to undergo a breast augmentation. An example of this concept was indicated through a comment which expressed that undergoing an augmentation was the: "Best decision I have made. It has improved my mental health drastically!” [BA_FU2_18-30]. Participants were satisfied with the implants as well as the feel of their implants following augmentation. Exemplar quotations illustrating participants' satisfaction include: "I love my implants" [BA_FU2_18-30] and "I love everything about my implants, how they look and how they feel - they feel part of me" [BA_FU2_31-50].

\section{Medical Team}

Participants ( $\mathrm{n}=27)$ who underwent augmentation expressed their satisfaction regarding the medical team, especially the surgeon as well. Comments revealing participants' satisfaction with their surgeons were: "Surgeon's work was impeccable ... recommends him to everyone" [BA_FU1_31-50] and "Surgeon did a sensational job of making the breast implants look very natural" [BA_FU2_31-50]. Further women said that their "surgeon is awesome.", "Excellent surgeon." and "I would recommend my surgeon any day" [BA_FU2_31-50].

\section{Post-Operative Appearance}

The comments also indicated that participants $(n=9)$ were grateful for their image and the look of the breasts after breast augmentation. Exemplar quotations that indicated this concept were: "My breasts feel fantastically amazing and give me the womanly figure that God forgot to." [BA_FU1_31-50] and "I am extremely happy with my augmentation and lift. They are very natural looking and feeling" [BA_FU2_18-30]. Participants also expressed their satisfaction with the post-operative appearance by reporting that breasts are " ... So natural looking. No one has noticed they are fake until they touch them" [BA_FU2_18-30].

\section{Dissatisfaction with Breast Augmentation}

The Overall Outcome of the Surgery and Implants

Some participants $(n=40)$ described that they were dissatisfied with the overall outcome of the breast 
augmentation procedure for various reasons. Some were unhappy about the decision made to undergo an augmentation procedure. A comment that indicated this concept was: "Regretting it. I've had 2 revisions" [BA_FU1_1830]. Participants further revealed that not receiving the desired look of the breast following surgery was disappointing and this was psychologically challenging. A comment that expressed this was: “... This has not been a pleasant experience! There has been a lot of recovery time with the end result, not ideal! [sic]" [BA_FU1_31-50].

Participants also indicated their dissatisfaction with breast implants following breast augmentation. Some participants reported experiencing leaking of implants, infection, rupturing and having to replace implants, or concerns with textured implants. An exemplar quote indicating this was:

"After the silicone was removed (scraped out), this breast has never looked the same. Second time around; rupture on right breast again but not silicone this time." [BA_FU2_51-71]

\section{Post-Operative Appearance}

The study also identified comments on how participants $(n=47)$ are dissatisfied with the appearance of the breast/ breasts after surgery. It was revealed that participants had concerns regarding hollowing and scarring present on their breasts. For instance, it was revealed that “ ... the original implants had to be removed and surgery redone 3 months later. This resulted in more scaring [sic], which is not pleasant" [BA_FU2 _31-50]. Participants also reported that they are dissatisfied with the shape of the breasts following surgery: "My left breast I hate it's a completely different height and shape" [BA_FU2_18-30].

\section{The Medical Team}

Participants $(n=11)$ expressed their dissatisfaction with the medical team, especially surgeons, at their sites of surgery. Surgeons' performances were a common theme that was present which impacted upon the overall satisfaction with the surgical outcome: "I am extremely unhappy with my surgeon and the outcome." [BA_FU2_31-50], or the participants did not receive appropriate follow up care: "Not happy with the aftercare from surgeon." [BA_FU2_31-50] and surgeons were being "dismissive, unsupportive" [BA_FU2_51-71].

\section{Complications and Breast Symptoms \\ Post-Operative Complications}

Participants $(n=61)$ mainly revealed that they experience post-operative complications such as pain, tightness, and loss of sensation in the operated breasts, which they are dissatisfied with. Many reported that pain in the operated breast occurred with menstruation and during daily activities. For instance, it was indicated that “ ... breast pain is severe during my menstruation. It never was before my implants" [BA_FU2_31-50]. Another example that indicated this concept was: "My left always seems to have pain when driving or sleeping" [BA_FU2_18-30]. Tightness and lack of sensation were also reported by participants following breast augmentation at both follow up year 1 and 2. For instance, it was indicated that: "I have had chronic tightness and problems with my left breast implant since the first surgery. The left implant dislodged, and I had a second surgery" [BA_FU2_18-30]. Another patient reported: "I still don't have sensation on both breast" [BA_FU2_31-50] at follow up year 2.

\section{Short-Term Complications}

Participants further reported that they experienced shortterm complications such as infection of their implants and developing hematoma $(n=37)$ following breast augmentation. An exemplar quotation indicating these complications was: "Implants were not good, previously had an infection that were removed" [BA_FU1_31-50]. Presence of hematoma also seems to be a common complication in participants as well. A comment that illustrated this dissatisfaction with complications was: "I had a hematoma in my LB [left breast, sic] after surgery and it hasn't ever been right" [BA_FU2_31-50].

\section{Long-Term Complications}

Participants revealed that they experience complications such as rippling, device malposition, capsular contracture, rupturing and cyst formation long term since surgery $(n=31)$. One participant stated that: “ ... weird shape with rippling and deformed" [BA_FU2_31-50]. Some participants indicated that moving of the breast implant causes discomfort and requires surgery for correction. For instance, it was revealed that: "I had revision surgery ... as one of my implants moved considerably ... wish I knew more of the risk of the implants moving as it was not a great experience" [BA_FU1_31-50].

Participants seemed to be concerned about capsular contracture, which also appears to affect their satisfaction following breast augmentation: "I noticed I started to get 
a capsular contracture ... I am still not satisfied with the results" [BA_FU2_18-30]. Rupturing of the implants was also reported by a few participants, they particularly expressed " ... the right breast has never been the same after the initial silicone rupture" [BA_FU2_51-71]. Further, participants also commented on having "Cyst on $\mathrm{RB}$ [right breast, sic] sometimes can get a bit sensitive but no pain" [BA_FU2_31-50].

\section{Breast Symptoms}

Participants reported experiencing various breast symptoms following breast augmentation $(n=48)$. These include having firm breasts, hardening, numbness, lumps on the breasts, loss of nipple sensitivity and soreness. The dissatisfaction was indicated by stating that: "I was surprised and very disappointed to lose $99 \%$ of nipple sensitivity" [BA_FU2_51-71]. Some participants reported experiencing multiple breast symptoms at the same time revealing that: "the underside of my breasts still fell a little numb. My nipple at times can get sensitive and sore" [BA_FU2_18-30].

\section{Other Comments}

This theme included comments regarding the BREAST-Q IS $(n=3)$, breast cancer $(n=1)$, cost of breast device surgery $(n=4)$, education regarding breast implants and surgery $(\mathrm{n}=4)$, breast device surgery and pregnancy $(n=4)$ as well as comments that do not relate to surgical outcomes $(\mathrm{n}=7)$.

Participants provided comments on how various aspects of the BREAST-Q IS can be improved $(n=3)$. These include suggestions to add more questions or to make the questions clearer so that they could easily be understood. For instance, it was suggested that "it would be great if the questionnaire covers the firmness level check" [BA_FU2_31-50]. The comments demonstrated that participants were mainly concerned about the cost of breast device surgeries and that they had not been more informed about the expected outcomes of breast device surgeries.

Comments also indicated that receiving more information regarding revision surgery was important. For instance, it was stated that: “ ... if I knew I would have to have surgery a second time so close to my original procedure I would not have got implants" [BA_FU1_3150]. Participants revealed that they were unhappy with the final outcome compared to the cost they spent. Participants expressed their frustration by stating that: "It's only been 2 years and even after a year I have been upset with how my boobs look for the \$\$\$ I spent. Now looking to have them fixed" [BA_FU2_18-30]. Participants also indicated that they were unable to undergo a surgery for a second time because they are simply unable to "afford the surgery" [BA_FU2_31-50].

In addition, participants indicated that pregnancy can impact on the outcomes following breast augmentation. For instance, the inability to breastfeed following augmentation was revealed as: "I just recently had a baby and am still really pleased with how my breasts have held up after pregnancy, although I was unable to breastfeed" [BA_FU2_31-50]. Further, participants also utilized the free comment section to express concerns that were not directly related to outcomes of their surgery. An exemplar quote indicating this was that: "In the past 4 months I've experienced significant autoimmune flare ups (existing) ankylosing spondylitis knee and back arthritis and Chrons [sic]" [BA_FU2_18-30].

From the themes discovered from PROMs data, two emerging issues among participants undergoing breast augmentation were identified. These were Breast Implant Illness (BII) and rippling of the breast implants following surgery.

Comments revealed that participants reported a variety of symptoms and complications and have concluded that they occurred as a result of their breast implants and are therefore due to BII. For instance, it was stated that: "I have been experiencing extreme fatigue and joint pain and I wonder if it's related to my breast implants? I read about breast implant illness" [BA_FU2_31-50]. Another participants explained:

"I became really sick due to my implants (constant night sweats, horrible body odour, digestive issues, acne) and the worst ... so much brain fog to the point where I couldn't keep up with basic conversation ..." [BA_FU2_18-30]

The majority $(\mathrm{n}=18)$ of the comments under the longterm complication theme were related to rippling. Given, the significant number of comments present, rippling was identified as another emerging issue identified was rippling of the breast implants. For instance, it was stated that:

"I found ripple under that breast which my doctor told me to massage. It is still there, 4 months later. I am still massaging it. Now the issue is arising on the other side. Not sure if this normal.” [BA_FU2_31-50] 
Another quotation further indicated the extent to which rippling can impact a person's self-esteem:

"My mental health has declined as my confidence has been shattered, I'm still aiming to compete at some stage in fitness competitions, however, I now feel embarrassed to even wear anything low cut on a daily basis, as the rippling is exceptionally obvious, even when wearing a bra." [BA_FU2_31-50]

Participants also revealed that rippling becomes visible, leading to appearance issues. For instance, it was indicated that:

"The edges-they are rippled, and I can feel the implants edges under my arm when I lay or sometimes even move my arms forward or down to my sides. The rippling is obvious to see, and I have to be careful of my choices of swimwear/clothing."[BA_FU2_31-50]

\section{Discussion}

In this study, a sample of participants' comments to the open-ended question of the BREAST-Q IS collected by the ABDR were analyzed. Content analysis led to the 268 comments being grouped into four themes: satisfaction with breast augmentation, dissatisfaction with breast augmentation, complications and breast symptoms and other comments. Among these findings, rippling of the implants and BII were identified as emerging issues in the population studied. Our study illustrates that the open-ended question of the BREAST-Q IS offers a broad range of insights into participants' experiences after breast augmentation.

This is the first time that observations have been used in monitoring the performance of breast device surgery. Traditionally, the outcome of breast device surgeries was evaluated by surgeons; however, patients' perspectives of their own experiences of breast devices can provide arguably a more important end-user perspective. ${ }^{20}$ In breast device surgery, validated PROMs such as the BREAST-Q are commonly used to assess surgical outcomes. Although the BREAST-Q gives detailed data across a number of domains, the disadvantage is the time required to complete it. In comparison, the BREAST-Q IS is shorter, designed to be used in a registry setting, across a large number of patients and completed in a few minutes. Together with the quantitative PROMs data from the BREAST-Q IS module, these findings can be used by the registry in monitoring the performance of breast implants and for detecting emerging issues.
This study extends on previous knowledge of patients' satisfaction, ${ }^{20}$ by providing subjective perspectives and further highlighting the various aspects of breast augmentation that led to satisfaction. In healthcare, satisfaction can be considered as the extent to which the participants feel they have received high-quality healthcare. ${ }^{21}$ In our study, comments provided by participants undergoing augmentation indicated their satisfaction related to the aesthetics of the breast after surgery, including having the desired implant shape, size and appearance of their breasts. Our findings show that satisfaction also extended to other elements of the experience of surgery, with participants also indicating that they were grateful towards the surgeon for giving them the expected results, and for the delivery of advice and after-care. This is similar to findings of a qualitative study which showed that surgeons are caring, reassuring, understood patients' needs and concerns and provided adequate follow-up. Further, it was indicated that patient-physician relationship provides patients confidence to undergo their surgeries. ${ }^{22}$

Dissatisfaction related to various aspects of breast augmentation surgery, such as complications and end appearance of the breast, and a sizable number of participants commented on the lack of communication and follow up by surgeons. ${ }^{23}$ One potential role for the ABDR's PROMs data is as feedback to the clinicians on their performance. While the ABDR PROM questions can improve feedback comparing clinicians' performance, deidentified comments provide a richer picture of the patient experience. These deidentified comments could at some point in the future be used by clinicians to monitor their own performance, and if necessary, can help improve clinician-to-patient interaction. In addition, the findings provide first-hand experiences of participants and highlight aspects that can further improve in existing breast augmentation performance and care.

The study also identified two emerging issues among participants' responses to the open-ended question: rippling and BII. Unlike conventional and recognized emerging health issues, emerging issues are those characterized by low or non-existent scientific knowledge, high levels of uncertainty and different levels of acceptability by the relevant authorities and exposed populations. ${ }^{24}$ Although these issues are already well-known issues in breast augmentation surgery, ${ }^{25-27}$ this shows us the capacity of the open-ended question to identify which issues patients consider important to them or were not directly enquired about in the BREASTQ IS. Allowing patients to self-report concerns about BII 
through open-ended questions of the BREAST-Q IS allows us to listen to our participants' concerns without the risk of potentially precipitating breast implant anxiety. ${ }^{28}$ Further, ongoing analysis of participants allows us to monitor for other new or emerging concerns as a means of identifying previously unrecognized potential adverse effects of implants or complications that are not enquired about through the data collection form. Currently, the ABDR has developed a new version of the data collection form includes a question on BII which will be implemented in the near future.

Strengths of this study include the broad inclusion criteria and large number of responses, which were obtained through the routine data collection undertaken by the registry. This offers a broad insight into participants' experience than would have been possible through focus groups or interviews, although the comments are generally brief. Random selection of the sample ensured that selection bias was minimized during data collection. The use of conventional content analysis ensured that the findings directly reflect patients' personal experiences of breast device surgeries.

Limitations include that due to the qualitative nature of this study, the sample was selected randomly from the dataset, the sample comprises comments only from those individuals who chose to respond, therefore does not reflect the entire population enrolled in the registry, which may impact the generalizability of results. The open-ended question is not a mandatory field in the PROM; therefore, patients who are mostly either satisfied or dissatisfied with their end results may have been more likely to respond. The brevity of the responses also means that we lack the opportunity for a nuanced interpretation that may come with focus groups or interviews. Also, the data only reflect one timepoint, that do not give a longitudinal picture of the patient experience.

\section{Conclusion}

This study is the first to analyze participants' responses to the open-ended question of the BREAST-Q IS in the ABDR. Themes included satisfaction, dissatisfaction, complications and breast symptoms and other comments. Emerging issues identified included rippling and BII. This study provides insights into patient experiences and emerging issues in patients undergoing breast augmentation procedures, in addition to the growing literature of using PROMs in the breast device surgery field.

\section{Acknowledgments}

We would like to thank all the participants who provided answers to the questions of the PROM as well as the ABDR staff who assisted with data extraction.

\section{Author Contributions}

All authors made a significant contribution to the work reported, where that was in relation to the concept $(\mathrm{IH})$, design (IH, RR, PG), execution (IH, RJ), acquisition of the data (PG), analysis (RJ) and interpretation, or in all other areas including drafting (RJ), writing (RJ), revising (IH, RR, PG, EE) or critically reviewing this manuscript (IH, RR, PG, EE). All authors have agreed on the journal to while the article will be submitted; any significant changes made at the proofing stage and agreed on all versions of the manuscript before submission. All authors agreed to take responsibility and accountability for the contents on the manuscript, in accordance with the International Committee of Medical Journal Editors authorship guidelines.

\section{Funding}

The Australian Breast Device Registry is funded by the Australian Department of Health.

\section{Disclosure}

The authors declared no potential conflict of interest with respect to research, authorship and publications of this article.

\section{References}

1. Hopper I, Parker E, Pellegrini B, Mulvany C, Pace M, Ahern S, Earnest A, Cooter RD, Farrell G, Elder E, Moore CM, McNeil JJ on behalf of the ABDR. The Australian Breast Device Registry 2018 Annual Report. Monash University, Department of Epidemiology and Preventive Medicine, October 2019 Report No 3, 68 pages.

2. Caouette-Laberge L, Borsuk D. Congenital anomalies of the breast. Semin Plast Surg. 2013;27(1):36-41. doi:10.1055/s-0033-1343995

3. Hopper I, Best RL, McNeil JJ, et al. Pilot for the Australian Breast Device Registry (ABDR): a national opt-out clinical quality registry for breast device surgery. BMJ Open. 2017;7(12):1-7. doi:10.1136/ bmjopen-2017-017778

4. Deva AK, Cuss A, Magnusson M, Cooter R. The "game of implants": a perspective on the crisis-prone history of breast implants. Aesthet Surg J. 2019;39(Suppl_1):S55-S65. doi:10.1093/asj/sjy310

5. Mitry MA, Sogani J, Sutton EJ, et al. Rare cancer on the rise: an educational review of breast implant-associated anaplastic large cell lymphoma. J Breast Imaging. 2020;2(4):398-407. doi:10.1093/jbi/wbaa041

6. Australian Government. Department of Health and Ageing. Therapeutic Goods Administration. Australian regulatory guidelines for medical devices (ARGMD). 2011.

7. $\mathrm{Ng} \mathrm{S}$, Kirkman M, Fisher J, et al. Establishing the acceptability of a brief patient reported outcome measure and feasibility of implementing it in a breast device registry - a qualitative study. $J$ Patient Rep Outcomes. 2019;3(1):63. doi:10.1186/s41687-019-0152-z 
8. Ibrahim AM, Ayeni OA, Hughes KB, Lee BT, Slavin SA, Lin SJ. Acellular dermal matrices in breast surgery: a comprehensive review. Ann Plast Surg. 2013;70(6):732-738. doi:10.1097/ SAP.0b013e31824b3d30

9. Hallberg H, Rafnsdottir S, Selvaggi G, et al. Benefits and risks with acellular dermal matrix (ADM) and mesh support in immediate breast reconstruction: a systematic review and meta-analysis. $J$ Plast Surg Hand Surg. 2018;52(3):130-147. doi:10.1080/ 2000656X.2017.1419141

10. Ng S, Parker E, Pusic A, et al. Lessons learned in implementing Patient Reported Outcome Measures (PROMs) in the Australian Breast Device Registry (ABDR). Aesthet Surg J. 2020;42:sjaa376. doi:10.1093/asj/sjaa376

11. Cella D, Hahn E, Jensen S, et al. Patient-reported outcomes in performance measurement. 2015.

12. Ahern S, Ruseckaite R, Ackerman IN. Collecting patient-reported outcome measures. Intern Med J. 2017;47(12):1454-1457. doi:10.1111/imj.13633

13. Weldring T, Smith SM. Patient-Reported Outcomes (PROs) and Patient-Reported Outcome Measures (PROMs). Health Services Insights. 2013;6(1):61-68. doi:10.4137/HSI.S11093

14. Boyce MB, Browne JP. Does providing feedback on patient-reported outcomes to healthcare professionals result in better outcomes for patients? A systematic review. Qual Life Res. 2013;22(9):2265-2278. doi:10.1007/s11136-013-0390-0

15. Bradshaw C, Atkinson S, Doody O. Employing a qualitative description approach in health care research. SAGE. 2017;4:1-8.

16. Hsieh HF, Shannon SE. Three approaches to qualitative content analysis. Qual Health Res. 2005;15(9):1277-1288. doi:10.1177/ 1049732305276687

17. Elo S, Kyngas H. The qualitative content analysis process. $J A d v$ Nurs. 2008;62(1):107-115. doi:10.1111/j.1365-2648.2007.04569.x

18. Vaismoradi M, Turunen $\mathrm{H}$, Bondas T. Content analysis and thematic analysis: implications for conducting a qualitative descriptive study. Nurs Health Sci. 2013;15(3):398-405. doi:10.11111/nhs. 12048

19. Tong A, Sainsbury P, Craig J. Consolidated criteria for reporting qualitative research (COREQ): a 32-item checklist for interviews and focus groups.. Int J Qual Health Care. 2007;19(6):349-357. doi:10.1093/intqhe/mzm042
20. Morley R, Leech T. Optimal assessment tools in assessing breast surgery: patient reported outcome measures (PROMs) vs. objective measures. Gland Surg. 2019;8(4):416-424. doi:10.21037/ gs.2019.02.04

21. Chow A, Mayer EK, Darzi AW, Athanasiou T. Patient-reported outcome measures: the importance of patient satisfaction in surgery. Surgery. 2009;146(3):435-443. doi:10.1016/j.surg.2009.03.019

22. Klassen AF, Pusic AL, Scott A, Klok J, Cano SJ. Satisfaction and quality of life in women who undergo breast surgery: a qualitative study. BMC Womens Health. 2009;9:11. doi:10.1186/1472-6874-9-11

23. Sarwer DB, LaRossa D, Bartlett SP, Low DW, Bucky LP, Whitaker LA. Body image concerns of breast augmentation patients. Plast Reconstr Surg. 2003;112(1):83-90. doi:10.1097/01. PRS.0000066005.07796.51

24. Benoit Roig FB. Management of emerging public health issues and risks. In: Benoit Roig KW, Thireau V, editors. Multidisciplinary Approaches to the Changing Environment. Oxford, UK: Elsevier Science and Technology; 2019.

25. Adidharma W, Latack KR, Colohan SM, Morrison SD, Cederna PS. Breast implant illness: are social media and the internet worrying patients sick? Plast Reconstr Surg. 2020;145(1):225e-227e. doi:10.1097/PRS.0000000000006361

26. Diaz JF. Review of 494 consecutive breast augmentation patients: system to improve patient outcomes and satisfaction. Plast Reconstr Surg Glob Open. 2017;5(10):e1526. doi:10.1097/GOX.00000 00000001526

27. Pantelides NM, Srinivasan JR. Rippling following breast augmentation or reconstruction: aetiology, emerging treatment options and a novel classification of severity. Aesthetic Plast Surg. 2018;42 (4):980-985. doi:10.1007/s00266-018-1117-y

28. Jewell ML, Jewell HL. breast implant-associated illness: medicine by belief, so says Dr. Google. Aesthet Surg J. 2019;39(4):NP87-NP89. doi:10.1093/asj/sjz007

\section{Publish your work in this journal}

Patient Related Outcome Measures is an international, peer-reviewed, open access journal focusing on treatment outcomes specifically relevant to patients. All aspects of patient care are addressed within the journal and practitioners from all disciplines are invited to submit their work as well as healthcare researchers and patient support groups.
The manuscript management system is completely online and includes a very quick and fair peer-review system. Visit http://www. dovepress.com/testimonials.php to read real quotes from published authors. 